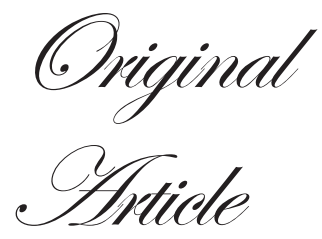

\title{
Alterations in intestinal permeability and endotoxemia in severe acute pancreatitis
}

\author{
Manik Sharma, ${ }^{1}$ Vikas Sachdev, ${ }^{1}$ Namrata Singh, ${ }^{1}$ Payal Bhardwaj, ${ }^{1}$ \\ Anuradha Pal, ${ }^{2}$ Suman Kapur, ${ }^{2}$ Anoop Saraya ${ }^{1}$
}

\section{ABSTRACT}

Background: Intestinal permeability increases early in the course of acute pancreatitis and is

Department of Gastroenterology and Human Nutrition Unit, ${ }^{1}$ All India Institute of Medical Sciences, New Delhi-110029 Center for Biotechnology, Biological Sciences Group, ${ }^{2}$

Birla Institute of Technology and Science, Pilani, Rajasthan, India

\section{Correspondence:}

Dr. Anoop Saraya

Email:ansaraya@yahoo.com associated with sepsis and organ failure.

Aim: To evaluate the intestinal permeability (IP) and anti-endotoxin antibodies immunoglobulin G and A (AEA IgG and A) in severe acute pancreatitis (SAP) as compared to healthy controls and determine their significance in relation to various complications of SAP.

Methods: IP was measured by urinary lactulose/mannitol (LM) excretion ratio and antiendotoxin antibodies by Endocab ELISA kit at days one and seven of admission (D1 and D7). Results: Thirty one patients of SAP [mean age (42.0 \pm 15.8$)$ years, APACHE II scores $(8.8 \pm 5.4)$ and CT severity index (6.4 \pm 2.0$)$ ] were included in this study. Infected pancreatic necrosis was detected in $13(42 \%)$ patients of whom three died. Six died of persistent organ failure. Median values of LM ratio at D1 and D7 were similar to those in healthy controls. Patients experiencing complications [organ failure $(4 / 9,44 \%)$, infected pancreatic necrosis $(5 / 10,50 \%)$ and death (1/ $2,50 \%)$ ] manifested a substantial increase in their intestinal permeability at D7. Anti-endotoxin antibodies $\operatorname{IgG}$ were lower $(\mathrm{p}=0.003)$ in patients than the controls at admission. AEA IgG were lower $(\mathrm{p}=0.03)$ in non-survivors as compared to survivors at D7.

Conclusion: Patients experiencing complications of severe acute pancreatitis showed an increase in intestinal permeability. Higher endotoxemia predicted poor outcome in severe acute pancreatitis.

KEYWORDS: Acute pancreatitis, complications, intestinal permeability, endotoxemia

\section{Introduction}

Gut mucosa is an effective barrier against spread of toxins. It has been shown in experimental animal models that the intestinal barrier is compromised in the early phase of acute pancreatitis (AP) resulting in translocation of enteric bacteria across the gut. ${ }^{1,2}$ Likewise, human studies have also demonstrated that intestinal permeability (IP) increases early in severe acute pancreatitis (SAP). ${ }^{3-5}$ The major mechanism that has been postulated for increased IP is splanchnic hypoperfusion and ischemia. The cause of splanchnic hypoperfusion is mainly due to hypovolemia. ${ }^{6}$ Increase in IP results in translocation of gram negative bacteria (GNB) through the lymphatics via the mesenteric nodes ${ }^{1,7} \mathrm{An}$ antibody response is stimulated against bacterial endotoxins comprising of the enterobacterial antigens attached to the gram negative bacterial cell wall. Previous studies involving patients with AP, have noted endotoxemia associated with systemic inflammatory response syndrome, multi-organ failure and high mortality. ${ }^{8-10}$ Hence this casecontrol study was undertaken to investigate the intestinal 
permeability and endotoxemia in patients with SAP and assess the relationship of these variables with various complications associated with them.

\section{Methods}

Study design

We organized our study as prospective case-control observational study.

\section{Patients and controls}

A total of 192 consecutive patient with acute pancreatitis and 15 healthy controls were included in this prospective study from January 2005 to December 2006. Of all these patients, 106 (55.2\%) had mild and $86(44.8 \%)$ had severe acute pancreatitis.

\section{Inclusion and exclusion criteria}

A total of 86 patients with SAP were enrolled for evaluation. Out of these, 55 patients were excluded from the study for the following reasons: 1 ) presence of renal failure at disease onset or at presentation to the hospital $(n=22) ; 2)$ patients who presented with proven or suspected infected pancreatic necrosis ( $\mathrm{n}=12)$; 3) patients who had been instituted on enteral nutrition prior to inclusion in the study $(\mathrm{n}=18) ; 4)$ patients who did not consent enrolment $(n=2)$. Thirty one patients with severe AP formed the study group.

\section{Diagnosis of severe acute pancreatitis}

All consecutive patients of acute pancreatitis were included in the study. The diagnosis of acute pancreatitis was based on clinical features, raised amylase ( $>3$ times the normal) and evidence of acute pancreatitis on imaging studies i.e. abdominal ultrasonography (USG) and/or contrast enhanced computed tomography (CECT) scan of the abdomen.

\section{Severity of acute pancreatitis}

The disease severity of severe acute pancreatitis (SAP) was defined by the presence of at least one of the following criteria: i) failure of one or more organ as defined by the Atlanta classification; ${ }^{11}$ ii) an Acute Physiology and Chronic Health Evaluation II (APACHE II) score $>8$; and/or iii) a Computed
Tomography Severity Index (CTSI) of $>7$. Pancreatic necrosis was diagnosed when non-enhancing areas were discovered on a contrast enhanced CT scan of the pancreas. The extent of pancreatic necrosis was graded as $<30 \%, 30-50 \%$ and $>50 \%$. CTSI was calculated according to the protocol devised by Balthazar et al. ${ }^{12}$

\section{Management of severe acute pancreatitis}

All patients were treated in an intensive care unit initially on a nil oral regime along with analgesics, aggressive fluid resuscitation and supportive treatment. Antibiotics were prescribed only if a patient had infected pancreatic necrosis or when there was documented infection at one or more extrapancreatic sites. The antibiotics were chosen according to the culture and sensitivity report wherever available. Enteral feeding was started early and calorie intake was increased gradually from 500 to $2000 \mathrm{Kcal} /$ day until day 7 of feeding and then continued until the patient resumed a near normal diet. The enteral feed was prepared in the hospital kitchen and its tolerability was monitored by the treating physicians. In patients with organ failure, all possible organ support systems were utilized. Patients with biliary obstruction or cholangitis underwent an endoscopic retrograde cholangiography. All patients with infected pancreatic necrosis were treated initially with antibiotics, early enteral nutrition, organ support and percutaneous catheter drainage. Patients who did not improve despite maximal supportive management underwent open necrosectomy with lavage, usually 4 weeks after the onset of pancreatitis.

\section{Outcome measures}

The primary outcome measures of our study included changes in intestinal permeability and endotoxemia in patients with severe AP in comparison with healthy controls and their relation to various complications of SAP.

\section{Methodology for measurement of intestinal permeability}

Intestinal permeability was measured in all enrolled severe AP patients on day 1 (D1) of their admission with a repeat assessment on day 7 (D7). It was measured by means of the urinary lactulose-mannitol (LM) test. ${ }^{5,13,14}$ 
Lactulose-mannitol test: Patients received $50 \mathrm{~mL}$ of water containing 10 grams lactulose and 5 grams mannitol either orally or through a nasogastric tube. Urine was collected for 5 hours in a receptacle with $0.2 \mathrm{~mL}$ of $2 \%$ clorhexidine to avoid bacterial overgrowth. The total urinary volume was measured and two aliquots of urine $10 \mathrm{~mL}$ each, were immediately frozen to $-70^{\circ} \mathrm{C}$ until analysis.

Lactulose was estimated using Selivanoff's method for quantitating ketose sugars. ${ }^{13}$ Mannitol was estimated using the Corcoran and Page method. ${ }^{14}$ The ratio of above lactulose and mannitol values was thus computed and defined as the LM ratio. The methodology of these tests has been described in detail by Nagpal et al. ${ }^{5}$ The ratio of these disaccharide molecules was chosen for our study because they have the properties of an ideal substrate for measuring gut permeability, are inexpensive and their utility for the above purpose has been validated in previous studies. ${ }^{5}$

\section{Estimation of endotoxemia}

Endotoxemia, as measured by anti-endotoxin $\operatorname{IgG}$ and $\operatorname{IgM}$, was measured with the Endocab ELISA kit (Hycult Biotechnology, Netherlands). The assay is based on a solid-phase sandwich ELISA, and detects and quantifies antibodies against endotoxin. The test was conducted as per the manufacturer's instructions, and a drop in antibody levels was reported directly proportional to the severity of endotoxemia.

\section{Statistical analysis}

All data comprising of categorical and interval variables were computed and presented as frequencies, percentages, means and standard deviations. Independent student $t$ tests were performed to compare the difference between patients and control for interval variables. Pearson correlation coefficient was calculated to assess for associations between appropriate study variables. A p value of 0.05 (two tailed) was considered as statistical significant. The SPSS v18.0 statistical package was used for analysis.

\section{Results}

Thirty one patients were included in this study, of which six were subsequently excluded. Two patients died within 7 days of admission, one developed renal failure during the course of study and in three patients the day 7 urine samples were misplaced. Anti-endotoxin antibodies were measured in all patients except for the two patients who succumbed to their illness. Fifteen healthy physicians comprised the control group.

Mean age of the patients and controls were comparable at $42.0 \pm 15.8$ and $39.2 \pm 14.6$ years respectively. The median duration of pain experienced prior to admission lasted 9 days. The etiology was gall stones in 17 (54.8\%) and alcohol in 6 (19.3\%) patients respectively. Computed tomography was undertaken in 24 patients and 13 of them revealed $>30 \%$ necrosis. Seventeen patients $(54.8 \%)$ were in organ failure at admission. Respiratory failure was seen in 12 patients; renal failure in five and one patient experienced GI bleed and cardiovascular collapse. Surgery was conducted in two patients, while another seven were put on percutaneous catheter drainage. Overall 9 patients died during hospitalization, six due to persistent organ failure and three because of infected pancreatic necrosis (Table 1). Blood cultures were positive in 9 (29\%) patients. E. coli, Pseudomonas spp. and Klebsiella spp. were identified in two patients each, while one had an Acinetobacter spp. infection. Another two patients tested positive for de novo fungal infection.

Table 1: Baseline characteristics of all patients $(n=31)$ at admission

Days between onset and admission

- Mean \pm SD

$11.42 \pm 10.41$

- Median 9

- Range $1-42$

APACHE II (Admission)

- Mean \pm SD

- Median

$8.82 \pm 5.49$

- Range 8 $2-24$

APACHE II (Worst)

- Mean \pm SD $10.79 \pm 6.07$

- Median 10

- Range $0-24$

CT Severity Index

- Mean \pm SD

$6.42 \pm 2.0$

3-10

- Range $17(54.8 \%)$

Organ failure (at admission) n (\%)

$6(19.3 \%)$

Co-morbid problems (\%)

$13(41.9 \%)$

IPN n (\%)

$2.85 \pm 0.69$

Hospital stay (days)

- Mean \pm SD

$19.32 \pm 9.77$

- Range

7-55

Mortality n (\%)

$9(29 \%)$

APACHE II: Acute physiology and chronic health evaluation IPN: Infected Pancreatic Necrosis

CT: Computed Tomography

SD: Standard Deviation 
Intestinal permeability (IP) was measured at admission and repeated after 7 days. The median values of IP were similar in patients of severe acute pancreatitis as compared to controls both at day 1 and at day 7 of admission (Table 2). Subgroup analysis showed that $4 / 9(44 \%)$ of patients with organ failure, $5 / 10(50 \%)$ with infected pancreatic necrosis and one of the two patients who died, witnessed a 4-10 times increase in their IP values from the baseline. Of the five patients with infected pancreatic necrosis who showed an increase in IP, four developed persistent organ failure and one died. The extent of change in IP in those who developed infected necrosis, organ failure and subsequently died has been depicted in Figure 1. No significant correlation was found between APACHE II scores and change in intestinal permeability at day 7 ( $\mathrm{r}=0.14$, $\mathrm{p}=0.56)$.

Endotoxin antibodies ( $\mathrm{IgG})$ at admission were much lower in patients with severe acute pancreatitis as compared to healthy controls $(\mathrm{p}=0.003)$ (Table 3). The levels of endotoxin antibodies (IgG) at admission (mean 393.1 GMU/ml vs. 332.1 GMU/ml, p=0.56) and at day 7 (mean 499 GMU/ml vs. 207 GMU/ $\mathrm{ml}, \mathrm{p}=0.02$ ) were high among survivors than in non-survivors (Figure 2). Thus higher levels of endotoxemia during the course of disease predicted poor outcome. No definite correlation was found between intestinal permeability and endotoxemia either at admission or at day $7(\mathrm{r}=0.26, \mathrm{p}=0.56)$.

Table 2: Intestinal permeability on Day 1 and Day 7 of admission

\begin{tabular}{llll}
\hline & \multicolumn{2}{c}{ LM ratio } & p \\
& Median & Range & value \\
\hline Control $(\mathrm{n}=15)$ & 0.128 & $0.015-0.666$ & \\
Day 1 of Admission $(\mathrm{n}=31)$ & 0.09 & $0.019-0.6$ & $0.25 *$ \\
Day 7 of Admission $(\mathrm{n}=25)$ & 0.135 & $0.009-0.781$ & $0.59 * *$ \\
\hline *denotes $p$ value between controls and Day 1 values & \\
$* *$ denotes $p$ value between control and Day 7 values &
\end{tabular}

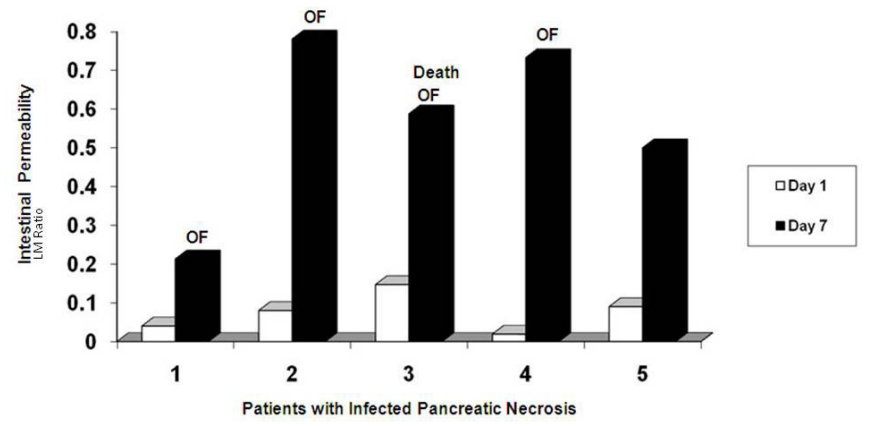

(Organ failure -OF)

Figure 1: Changes in IP in patients with infected pancreatic necrosis.

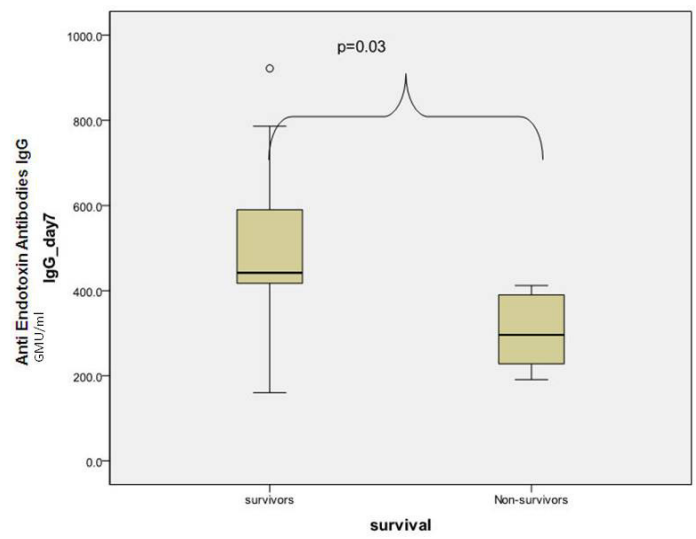

Line in the Box-plot graph expresses median values. Pvalue denotes significance between Day 7 values of Survivors and Non-

Figure 2: Box-plot graph of anti-endotoxin antibodies ( $\operatorname{IgG}$ ) at Day 7 of admission - survivors versus non-survivors.

Table 3: Anti Endotoxin Antibodies (EndoCab IgG and IgM) in severe acute pancreatitis.

\begin{tabular}{lllll}
\hline & \multicolumn{2}{c}{ IgG (GMU/ml) } & \multicolumn{2}{c}{ IgM (MMU/ml) } \\
& Day 1* & Day 7 & Day 1 & Day 7 \\
\hline Patients (n) & 31 & 29 & 31 & 29 \\
Median & $368^{*}$ & 412 & 80.2 & 76.3 \\
Range & $168-556$ & $160-922$ & $12.8-190$ & $28-172$ \\
Control (n) & 15 & & 15 & \\
Median & $520^{*}$ & & 92.6 & \\
Range & $194-960$ & & $60.6-168$ & \\
\hline
\end{tabular}

$* p=0.003$

\section{Discussion}

Over the natural course of severe acute pancreatitis, intestinal permeability has been found to increase within the first 72 hours and it correlates strongly with the clinical outcome of the patient. ${ }^{3,5,15}$ Juoven et al $^{16}$ suggested that after an initial increase, the intestinal permeability in a patient gets restored between day 8 and day 45 of the illness. It has also been shown by others that the increase in IP is gradual and by second week the values start decreasing but they take longer to normalize. ${ }^{4}$ In our study, we found that the intestinal permeability (LM ratio) at day 1 and day 7 of admission was no different when compared with controls, which is in contrast to previous studies. There are three possible explanations for this observation in our study: i) the median interval between the onset of pain and admission in our study was 9 days, thereby implying that the first measurement of intestinal permeability was made in the early second week of the illness, when most of the restitution of intestinal barrier would have already started. The main reason for delay in admission was due to late referral to our hospital (25\% of our patients were excluded from this 
study at the very outset, due to the above reason); ii) the intestinal permeability might have been restored by enteral nutrition instituted at the referring hospital, although our enrolment was stringently screened to exclude such patients; iii) a small sample size could have precluded us from reaching any meaningful conclusions. However, on subgroup analysis of those patients who had major complications of severe pancreatitis (organ failure, infected pancreatic necrosis and death), we found that 44 - 50\% of such patients witnessed an increase in their IP on day 7 as compared to their day 1 values. Though it can be argued that such complications of severe AP could have occurred because of the aggravation of the disease itself by the second week, rather than by change in intestinal permeability; we found no correlation between the worst APACHE II scores and intestinal permeability ( $\mathrm{r}=0.14, \mathrm{p}=0.56)$. Though the intestinal permeability did not show any remarkable difference between the patients and controls, anti-endotoxin antibodies ( $\operatorname{IgG}$ ) were significantly low in patients with severe pancreatitis as compared to controls, even in the second week of disease. This observation was congruent to the fact that these endotoxin antibodies are formed against the enterobacterial antigen of gram negative bacteria. Endotoxin antibodies were depleted in non-survivors, proving that they have impairment of systemic immunity and could not mount an immune response against the bacteria and fungi localizing across the compromised intestinal barrier. These results are similar to previous studies which had shown endotoxemia as a valid prognostic marker for various complications, like organ failure and mortality in severe acute pancreatitis. ${ }^{8-10,17-19}$ These reported findings also have an impact on the clinical management of such patients. Various clinical trials involving selective decontamination, oral antibiotics and enteral and immunonutrition have already been tried with varied efficacy. ${ }^{20-}$ ${ }^{25}$ Larger well designed trials assessing various combination strategies for preventing bacterial transmigration into pancreatic tissue are recommended for validating the above findings.

There were few limitations in our study. First, measurement of IP was done late into the second week of disease rather than at the onset of pain. Nevertheless, it helped us to validate the erstwhile understanding that intestinal permeability gets restored by second week as suggested previously and continues to improve thereafter. Also this observation indicates that any intervention aimed to regulate intestinal permeability has to be instituted in the initial one week and any effort later than this crucial period may not be meaningful. Second, the number of patients recruited in this study was small and the attrition was a consequence of a large number of patients being excluded due to our strict inclusion criteria. Third, intestinal permeability was not measured in mild acute pancreatitis (MAP). In patients with MAP, the disease resolves rapidly and often does not result in any systemic manifestations or pancreatic necrosis. Hence such patients do not show any significant changes in their intestinal permeability. Lastly, IP was not measured by other more sensitive techniques including high performance liquid chromatography (HPLC) or the high molecular weight polyethylene glycol (PEG) assay, as these were not available in our hospital at the time of this study.

In conclusion, intestinal permeability is considerably increased in patients who develop serious complications of severe acute pancreatitis including organ failure, infected pancreatic necrosis and death. Patients who do not survive have higher endotoxemia compared to those who withstand the onslaught of disease. Future trials with aggressive enteral therapy and locally acting antibiotics may help to assess the reduction in bacterial translocation and prevention of endotoxemia in severe AP.

\section{References}

1. Tarpila E, Nystrom PO, Franzen L Ihse I. Bacterial translocation during acute pancreatitis in rats. Eur J Surg. 1993;159:109-13.

2. Ryan CM, Schmidt J, Lewandrowski K, Compton CC, Rattner DW, Warshaw AL, et al. Gut macromolecular permeability in pancreatitis correlates with severity of disease in rats. Gastroenterology. 1993;104:890-5.

3. Ammori BJ, Leeder PC, King RF. Early increase in intestinal permeability in patients with severe acute pancreatitis: correlation with endotoxemia, organ failure and mortality. J Gastrointest Surg. 1999;3:252-62.

4. Penalva JC, Martínez J, Laveda R, Esteban A, Muñoz C, Sáez $\mathrm{J}$, et al. A study of intestinal permeability in relation to the inflammatory response and plasma endocab IgM levels in patients with acute pancreatitis. J Clin Gastroenterol . 2004;38:512-7.

5. Nagpal K, Minocha VR, Agrawal V, Kapur S. Evaluation of intestinal mucosal permeability function in patients with acute pancreatitis. Am J Surg. 2006;192:24-8.

6. Rahman SH, Ammori BJ, Holmfield J, Larvin M, McMahon MJ. Intestinal hypoperfusion contributes to gut barrier failure in severe acute pancreatitis. J Gastrointest Surg. 2003;7:26-35; discussion 35-6.

7. Runkel NS, Moody FG, Smith GS, Rodriguez LF, La Rocco MT, Miller TA. The role of gut in the development of sepsis in acute pancreatitis. J Surg Res. 1991;51:18-23.

8. Exley AR, Leese T, Holliday MP, Swann RA, Cohen J. Endotoxaemia and serum tumour necrosis factor as prognostic markers in severe acute pancreatitis. Gut. 1992;33:1126-8.

9. Windsor JA, Fearon KC, Ross JA, Barclay GR, Smyth E, Poxton 
I, et al .Role of serum endotoxin and antiendotoxin core antibody levels in predicting the development of multiple organ failure in acute pancreatitis. Br J Surg. 1993;80:1042-6.

10. Kivilaakso E, Valtonen VV, Malkamäki M, Palmu A, Schröder T, Nikki P, et al Endotoxaemia and acute pancreatitis: correlation between the severity of the disease and the anti-enterobacterial common antigen antibody titre. Gut. 1984;25:1065-70.

11. Bradley EL 3rd. A clinically based classification system for acute pancreatitis. Summary of the International Symposium on Acute Pancreatitis, Atlanta, Ga, September 11 through 13, 1992. Arch Surg. 1993;128:586-90.

12. Balthazar EJ, Robinson DL, Megibow AJ, Ranson JH. Acute pancreatitis: value of CT in establishing prognosis. Radiology. 1990; 174:331-6.

13. Burtis CA, Ashwon ER. Carbohydrates. In: Teitz NW, editor. Fundamentals of clinical chemistry. Philadelphia, PA: WB Saunders Co; 1987.p.426-30.

14. Corcoran AC, Page IH. Method for the determination of mannitol in plasma and urine. Fed Proc. 1946;5:130.

15. Liu H, Li W, Wang X, Li J, Yu W. Early gut mucosal dysfunction in patients with acute pancreatitis. Pancreas. 2008;36:192-6.

16. Juvonen PK, Alhava EM, Takala JA. Gut permeability in patients with acute pancreatitis. Scand J Gastroenterol. 2000;35:1314-8.

17. Martínez J, Palazón JM, Muñoz C, López M, Sánchez-Payá J, Laveda R, et al. Endotoxin and anti-endotoxin antibodies in the prognosis of acute pancreatitis. Rev Esp Enferm Dig. 2002;94:406-16.

18. Barclay GR. Endogenous endotoxin-core antibody (EndoCAb) as a marker of endotoxin exposure and prognostic indicator: a review. Prog Clin Biol Res. 1995;392:263-72.

19. Wig JD, Kochhar R, Ray JD, Krishna Rao DV, Gupta NM, Ganguly NK. Endotoemia predicts outcome in acute pancreatitis. J Clin Gastroenterol. 1998;26:121-4.

20. Gianotti L, Munds R, Gennari R, Pyles R, Alexander JW. Effect of different regimens of gut decontamination on bacterial translocation and mortality in experimental acute pancreatitis. Eur J Surg. 1995;161:85-92.

21. Luiten EJ, Hop WC, Lange JF, Bruining HA. Controlled clinical trial of selective decontamination for the treatment of severe acute pancreatitis. Ann Surg. 1995;222:57-65.

22. Kalambokis G, Tsianos EV. Endotoxaemia in the pathogenesis of cytopenias in liver cirrhosis. Could oral antibiotics raise blood counts? Med Hypotheses. 2011;76:105-9.

23. Targarona Modena J, Barreda Cevasco L, Arroyo Basto C, Orellana Vicuña A, Portanova Ramírez M. Total enteral nutrition as prophylactic therapy for pancreatic necrosis infection in severe acute pancreatitis. Pancreatology. 2006;6:58-64.

24. Souba WW, Klimberg VS, Hautamaki RD, Mendenhall WH, Bova FC, Howard RJ, et al. Oral Glutamine reduces bacterial translocation following abdominal radiation. J Surg Res. 1990;48:1-5.

25. Foitzik T, Kruschewski M, Kroesen AJ, Hotz HG, Eibl G, Buhr HJ. Does glutamine reduce bacterial translocation? A study in two animal models with impaired gut barrier. Int J Colorectal Dis. 1999;14:143-9. 